\title{
Transparency poor in academic medical research
}

$\mathrm{W}$ hen it comes to sharing the results of clinical trials, it seems academic medical centres are no more generous than pharmaceutical companies.

"Despite the ethical mandate and expressed values and mission of academic institutions, there is poor performance and noticeable variation in the dissemination of clinical trial results across leading academic medical centres," concluded the authors of a recent study in $B M J$.

This comes as no surprise to Dr. Ben Goldacre, an academic and science writer who cofounded the AllTrials campaign, which calls for all clinical trials to be registered and published. In a previous news article in CMAJ, Goldacre referred to the withholding of clinical trial results as research misconduct. And it is well known, he said, that many researchers in both academia and industry fail to share the findings of their work.

"But just because it's familiar, we shouldn't stop caring: this is a widespread cultural failing throughout medicine, and it's letting patients down," Goldacre wrote in an email, adding that change is unlikely without accountability.

"Audit is the most basic and useful tool we have in medicine to find out who is doing well. So, if individual researchers are performing badly, and failing to share trial results, then we need to look at them very carefully, ask them why they are failing, and help them learn from those who are performing well, as with anything in medicine."

The $B M J$ study looked at 4347 clinical trials conducted at 51 academic medical centres in the United States. Publication rates within two years of study completion at each institution ranged from $10.8 \%$ to $40.3 \%$. Reporting of results on ClinicalTrials.gov varied even more, ranging from $1.6 \%$ to $40.7 \%$. Overall, results from $66 \%$ of the clinical trials were eventually disseminated, though only $35.9 \%$ within two years of completion.

"I will tell you, personally, I was dis-

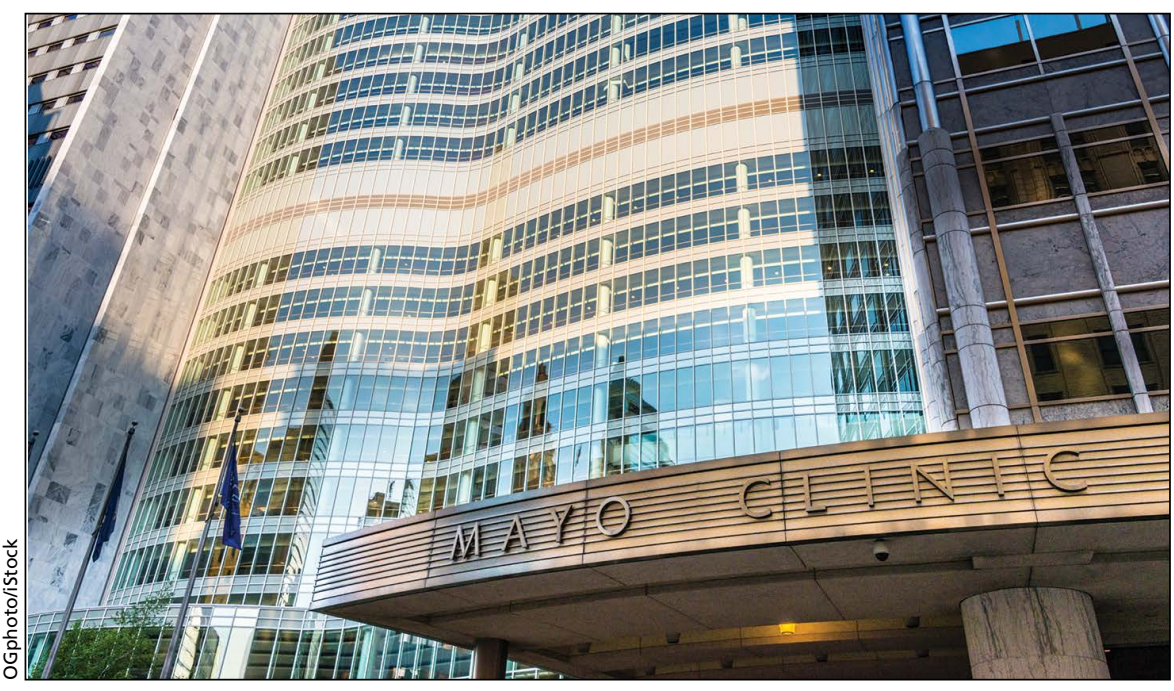

Rates of dissemination of clinical trial results at prestigious academic medical centres, such as the Mayo Clinic, vary widely across the United States.

appointed," Dr. Nihar Desai, one of the study's authors, wrote in an email. "Academic medical centres are committed to delivering the highest quality clinical care but also to advancing knowledge and training the next generation. I thought transparency and dissemination of clinical trials by our most prestigious institutions would be better than what we found."

Researchers may be reluctant to share results for many reasons. The findings could be unfavourable to funders, for example, or publication could lead to competition from other researchers. And though there are ethical imperatives and statutory requirements to share results, dissemination rates remain low because there are no enforcement mechanisms, said Desai, an assistant professor in the Yale School of Medicine.

"As such, investigators know that the requirements are requirements in name only. There have not been any ramifications for failing to disseminate results - either from academic centres, journals or funders."

A sense of urgency could be fostered, however, if there were greater incentives to publish, suggested Desai. What if, for instance, funders consid- ered previous performance on results dissemination in current funding decisions? Or medical journals considered it when accepting new manuscripts? Or academic centres incorporated it into the promotions process?

According to the Association of American Medical Colleges, there are several other issues to address to improve the timely reporting of research results and data quality. These include identifying a streamlined format for reporting results, developing a new system for results posting, and engaging patients and the public for input on how to create a user-friendly database.

"We support publishing timely results of clinical trials, and the format and venue should ensure that the information is meaningful, accurate and complete," Heather Pierce, the association's senior director of science policy and regulatory counsel, said in an email statement. "Academic institutions recognize the value of timely dissemination of clinical trial results that could impact patient care or medical practice and are actively engaged in discussions and activities to improve this process." — Roger Collier, CMAJ

CMAJ 2016. DOI:10.1503/cmaj.109-5254 